\title{
OSTEOARTHRITIS
}

\author{
Adrian Jones, Michael Doherty
}

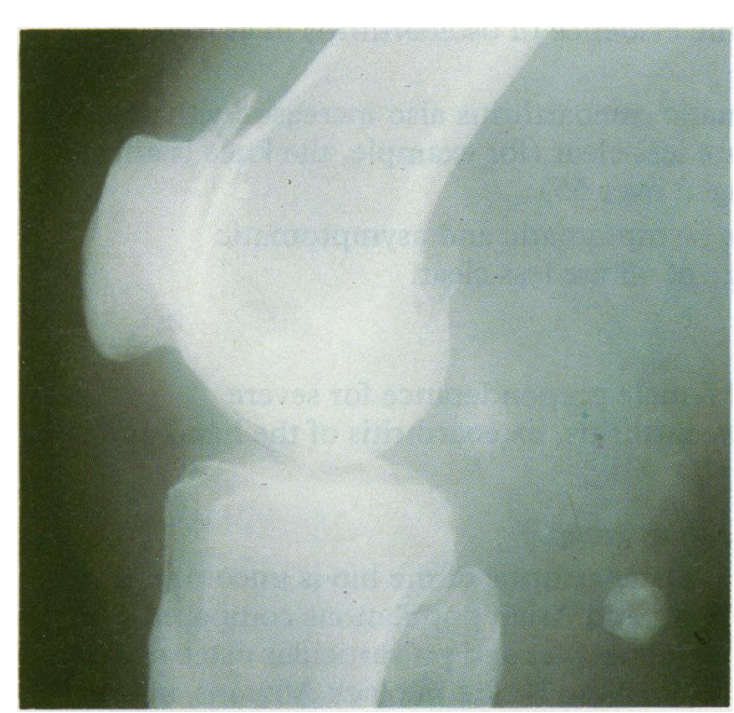

Radiograph of joint affected by osteoarthritis.
Osteoarthritis is the commonest condition to affect synovial joints, the single most important cause of locomotor disability, and a major challenge to health care. Previously considered as a degenerative disease that was an inevitable consequence of aging and trauma, osteoarthritis is now viewed as a metabolically dynamic, essentially reparative process that is increasingly amenable to treatment.

There is no generally accepted definition of osteoarthritis, but most would agree that pathologically it is a condition of synovial joints characterised by focal cartilage loss and an accompanying reparative bone response. Defining this in practice is less easy. Current definition of clinical cases hinges on detecting structural changes clinically or in radiographs. For many the plain radiograph remains the best means of assessment, with evidence of cartilage loss (joint space narrowing) and bone response (presence of osteophytes and sclerosis) being the main criteria. This definition, however, excludes joints with early minimal change, ignores tissues other than cartilage and bone, and omits consideration of biological consequences (symptoms and disability). There is often considerable discordance between structural change and clinical outcome; patients with apparent structural catastrophe may have few or no symptoms. Better understanding of the causes of symptoms and disability is currently a key challenge.

\section{Process of osteoarthritis}
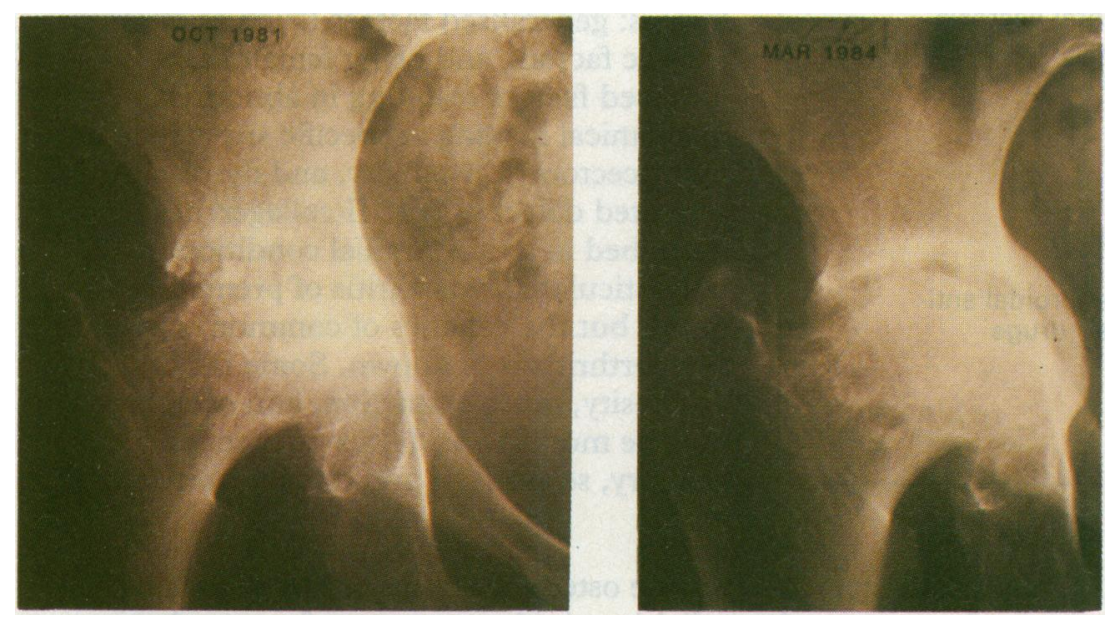

Example of healing osteoarthritis in hip joint.

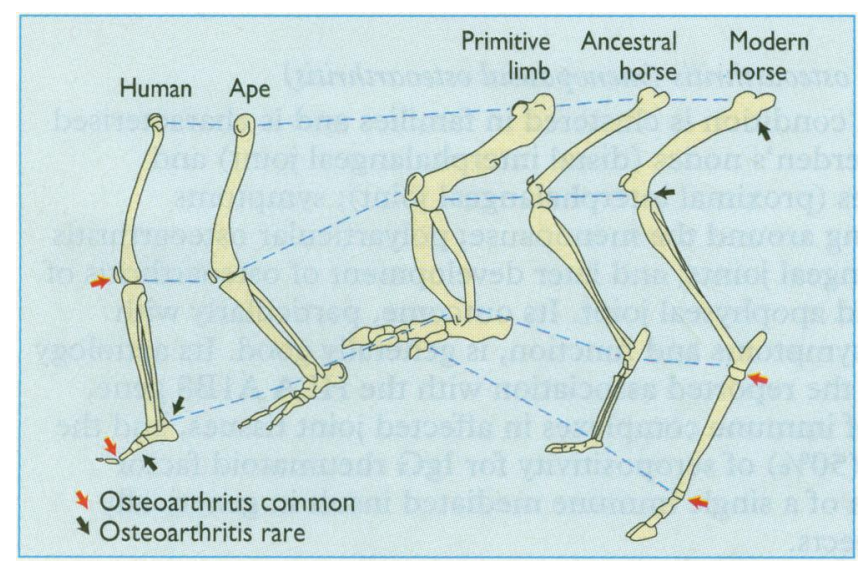

Association between osteoarthritis and evolution.
Observations about osteoarthritis have led to the suggestion that it is an aspect of the inherent repair process of synovial joints:

- Osteoarthritis has been present throughout evolution and is ubiquitous in humans and other vertebrates.

- Osteoarthritis is not simply the attrition of joint structures but is a metabolically active condition that shows a variable balance between anabolic and catabolic processes-at different stages there is increased activity in all joint tissues (cartilage, bone, synovium, capsule, and muscle)

- Osteoarthritis is common but is usually asymptomatic

- Occasionally, there is radiographic evidence of osteoarthritic joints "healing."

In most cases this slow but metabolically active process keeps pace with various triggering insults and is non-progressive, but sometimes it fails to compensate, resulting in joint failure (symptoms and disability). This interpretation partly explains the heterogeneity of osteoarthritis: various extrinsic and intrinsic insults cause different patterns of arthritis, and multiple constitutional and environmental factors modify response and outcome. Osteoarthritis targets specific joints, possibly those that have undergone recent evolutionary change in function (particularly relating to bipedal locomotion and precision grip) without yet adapting adequately. 


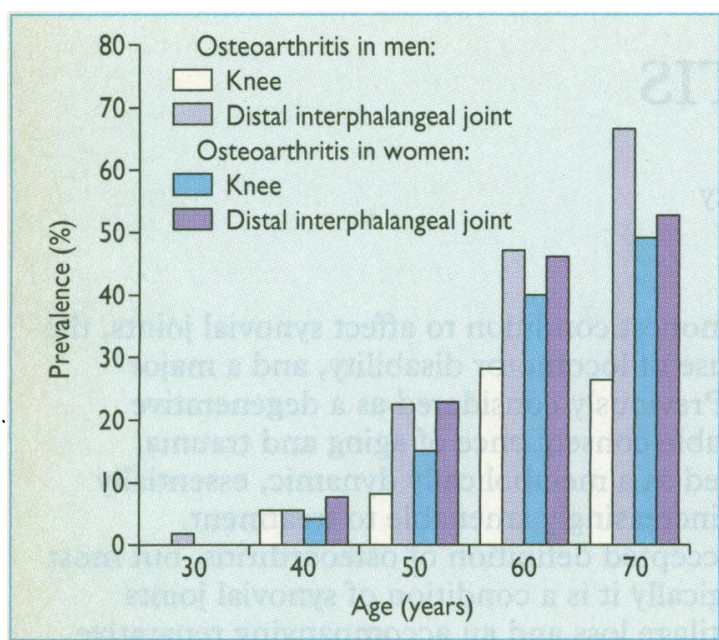

Prevalence of radiographic evidence of osteoarthritis in the population.

\section{Age}

Although not an inevitable consequence of aging, osteoarthritis is strongly related to age. This may represent cumulative insult to the joint, possibly aggravated by decline in neuromuscular function, or senescence of homoeostatic repair mechanisms. The consequence is a considerable medical burden and one that will increase with increasing numbers of elderly people in the British population. In general:

- Osteoarthritis is uncommon (and multiple joint osteoarthritis is rare) in people aged under 45

- Prevalence of osteoarthritis increases up to age 65 , when at least half of people have radiographic evidence of osteoarthritis in at least one joint group

- Prevalence of symptomatic osteoarthritis also increases with age, but data for this association are less clear (for example, the knee is affected in about $15 \%$ of people aged over 55 )

- Increases in prevalence (symptomatic and asymptomatic osteoarthritis) over the age of 65 are less clear.

\section{Sex}

There is a pronounced female preponderance for severe radiographic grades of osteoarthritis, osteoarthritis of the hand and knee, and symptoms.

\section{Ethnic group}

Osteoarthritis of the hip is uncommon in black and Asian populations compared with white people, and polyarticular osteoarthritis of the hand is rare in black Africans and Malaysians. This difference seems to reflect genetic rather than cultural differences.

\section{Putative risk factors for development and progression of hip and knee osteoarthritis Hip \\ Knee}

Development

Previous disease or trauma (such as Perthes' disease or slipped femoral epiphysis)

Acetabular dysplasia

Avascular necrosis

Non-gonococcal septic arthritis

Occupation (farming)

\section{Progression}

Superior pole pattern

Chondrocalcinosis (knee)

Obesity

Use of non-steroidal anti-

inflammatory drugs

"Protective" factors

Osteoporosis
Previous trauma (such as meniscectomy)

Distal femoral dysplasia Medial femoral necrosis Non-gonococcal septic arthritis Sex (female)

Pronouced varus

Sex (female)

Obesity

Use of non-steroidal antiinflammatory drugs

\section{Individual risk factors}

There are two main categories of such risk factors: generalised factors (such as obesity, genetic factors, and being female) and localised factors resulting in abnormal mechanical loading at specific sites (such as meniscectomy, instability, and dysplasia). An inherited defect in type II collagen has been described in a rare familial condition of polyarticular osteoarthritis of premature onset, but the genetics of common osteoarthritis is unknown. Some factors (such as obesity, muscle function, and occupation) may be modified and thus offer scope for primary, secondary, and tertiary prevention.

\section{Types of osteoarthritis}

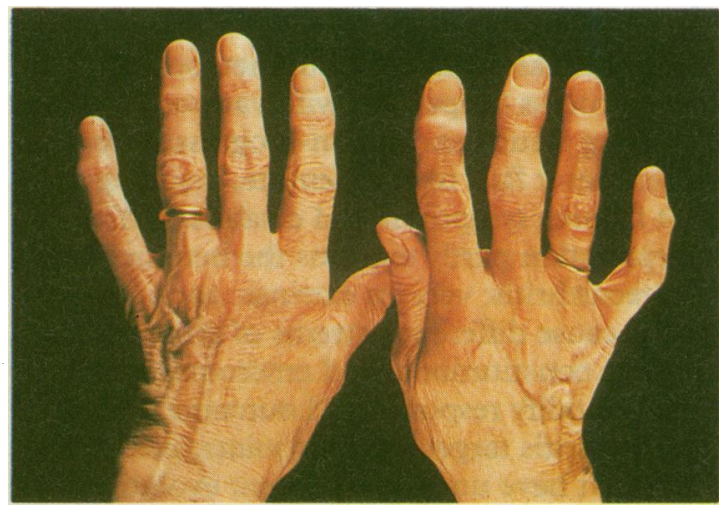

Nodal osteoarthritis.
Despite attempts to subdivide osteoarthritis according to various criteria, there are no sharp divisions in the spectrum of osteoarthritis. People may display different features at different sites and evolve from one "subset" to another. Nevertheless certain distinctions may be useful.

\section{Nodal generalised osteoarthritis (menopausal osteoarthritis)}

This common condition is clustered in families and is characterised by multiple Heberden's nodes (distal interphalangeal joint) and Bouchard's nodes (proximal interphalangeal joint); symptoms commonly starting around the menopause; polyarticular osteoarthritis of the interphalangeal joints; and later development of osteoarthritis of the knee, hip, and apophyseal joint. Its outcome, particularly with respect to hand symptoms and function, is generally good. Its aetiology is unknown, but the reported association with the HLA A1B8 gene, the occurrence of immune complexes in affected joint tissues, and the high prevalence $(50 \%)$ of seropositivity for IgG rheumatoid factor supports the idea of a single immune mediated insult in genetically predisposed subjects. 


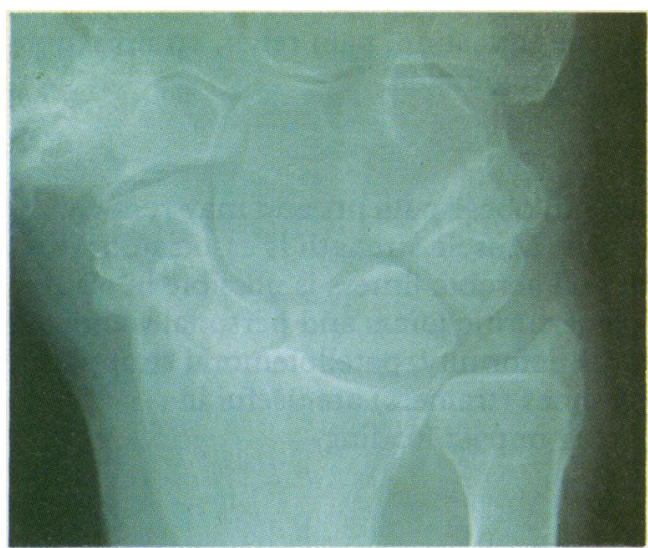

Radiograph of wrist showing chondrocalcinosis and arthropathy.

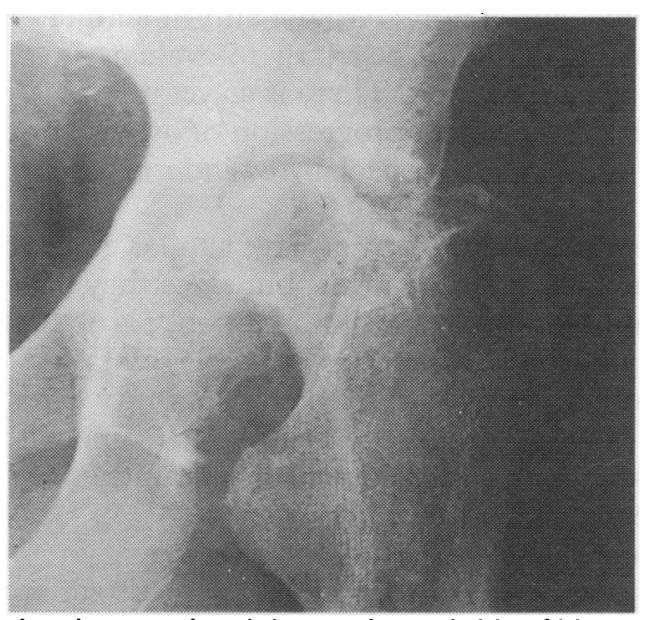

Apatite associated destructive arthritis of hip.

\section{Clinical features}

\section{Assessment of patients with osteoarthritis}

\section{Nature of pain}

Mechanical-related to use

Inflammatory-stiffness, pain aggravated by rest

Nocturnal-suggests intraosseous hypertension Sudden deterioration-consider sepsis, avascular necrosis, fracture, or crystal synovitis

Clinical examination

Periarticular or articular source of pain?

Generalised pain?-consider fibromyalgia

(test tender sites)

Presence of deformity?

Evidence of muscle wasting?

Local inflammation or effusion?

Generalised or localised osteoarthritis?

Joint locking

Orthopaedic referral probably appropriate

Weight

Potentially modifiable risk factor

Sleep disturbance

May be associated with fibromyalgia and depression

\section{Comorbid disease}

Modifies risk of treatment with non-steroida anti-inflammatory drugs and of surgery

May be evidence of disease associated with premature osteoarthritis (for example,

haemochromatosis or spondyloepiphyseal

dysplasia)

\section{Crystal associated osteoarthritis}

Calcium crystals, notably calcium pyrophosphate dihydrate and apatite, may deposit in cartilage as an isolated phenomenon but also commonly occur in osteoarthritic joints. In this context they probably arise via physicochemical changes accompanying the osteoarthritis process. Although these crystals may sometimes initiate inflammation (such as in acute pseudogout), they usually have no direct deleterious effects.

Osteoarthritis with deposition of calcium pyrophosphate dihydrate (pyrophosphate arthropathy) occurs predominantly in elderly women, principally affects the knee, and is associated with inflammation and widespread and pronouced radiographic changes (usually hypertrophic).

Apatite associated destructive arthritis-Although modest amounts of apatite are present in most osteoarthritic joints, large amounts occur in apatite associated destructive arthritis. This is almost totally confined to the hips, shoulders (Milwaukee shoulder), and knees of elderly women and has a poor outcome. There is typically rapid painful progression with large cool effusions, progressive instability, and atrophic radiographic changes. The differential diagnosis includes Charcot arthropathy, sepsis, and avascular necrosis.

\section{Osteoarthritis of premature onset}

Development of single joint osteoarthritis after severe trauma or alteration in joint biomechanics (for example, after meniscectomy or because of developmental abnormality) is not uncommon. However, premature onset (under the age of 50) in several joints should prompt consideration of predisposing metabolic, hormonal, or other causes. Conditions for which such osteoarthritis can be the presenting feature are rare, but some are amenable to correction (although existing osteoarthritis is usually unaffected). These conditions include:

- Haemochromatosis

- Ochronosis

- Acromegaly

- Spondyloepiphyseal dysplasia, epiphyseal dysplasia, and hereditary type II collagen defects

- Thiemann's disease

- Endemic osteoarthritis (normally rare but common in endemic areas).

The main clinical features of osteoarthritis are symptoms, functional impairment, and signs. There can be considerable discordance between these three. Pain may arise from several sites in and around an osteoarthritic joint. Suggested mechanisms include increased capsular and intraosseous pressure, subchondral microfracture, and enthesopathy or bursitis secondary to muscle weakness and structural alteration. Severity of pain and functional impairment are greatly influenced by personality, anxiety, depression, and daily activity.

Crepitus, bony enlargement, deformity, instability, and restricted movement may occur in any combination and predominantly reflect structural change. Varying degrees of synovitis (warmth, effusion, and synovial thickening) may be superimposed, and muscle weakness or wasting is usual. Periarticular sources of pain are often found, particularly at the knee and hip.

Assessment is directed at establishing the sources of symptoms in an individual patient. Determining the presence of osteoarthritis is not usually the problem - the usual question is whether osteoarthritis is relevant to the patient's complaints. The high prevalance of osteoarthritis in the general population means that comorbid conditions often exist. These include soft tissue lesions (enthesopathy and bursitis), fibromyalgia, gout, inflammatory arthritis, and sepsis. These require attention in their own right. Only an adequate history and examination can determine how much structural and inflammatory change is present and how much this contributes to a patient's overall problem. 


\section{Changes in lifestyle for patients with osteoarthritis}

\section{General measures}

Maintain optimal weight

Encourage activity and regular general exercise

Maintain positive approach

\section{Specific measures}

\section{Strengthening of local muscles}

Use of appropriate footwear and walking aids Pay attention to specific problems caused by disability (such as shopping, housework, and job)
The aims of treatment are patient education, pain relief, optimisation of function, and minimisation of progression.

\section{Biomechanical factors}

Weight reduction is desirable in obese patients and may reduce progression of knee osteoarthritis. Muscle strength is associated with disability. Increasing strength and aerobic fitness is possible in all age groups and is beneficial to osteoarthritic joints and personal wellbeing. Insoles to counteract knee varus deformity, patellofemoral strapping, walking sticks, and cushioned shoes (trainers) are useful in redistributing stress and reducing impact loading.

\section{Pain control}

Pain is the main reason why patients seek help. The current high use of non-steroidal anti-inflammatory drugs for osteoarthritis is probably mistaken. Adequate doses of analgesics such as paracetamol given regularly are usually adequate for most patients. Symptoms of osteoarthritis are often episodic, and if non-steroidal anti-inflammatory drugs are used their requirement should be regularly re-evaluated, especially in elderly patients who are at risk. There is as yet no convincing evidence that these drugs affect progression of osteoarthritis in humans, but hastening of progression (especially by indomethacin) has been suggested.

Topical non-steroidal anti-inflammatory drugs have similar efficacy to oral preparations in patients with osteoarthritis, but since they have fewer side effects they probably should be used more often, particularly for patients with just a few symptomatic, readily accessible joints. However, there remains substantial doubt as to their superiority over simple rubefacients. Several other symptomatic treatments (some with claimed chondroprotective action) are available in some countries or are under investigation. There is considerable interest in pharmacological manipulation of osteoarthritis (and exciting experimental data), but the precise role of such drugs in treatment is not yet defined.

\section{Disease modifying drugs}

The use of intra-articular corticosteroid injections for uncomplicated osteoarthritis is controversial and generally not recommended. They have a place, however, in treating patients with acute crystal associated synovitis or those who are unfit for or awaiting surgery. Their effect is generally transient. Similarly, the place of radioisotope synovectomy is unclear, although injections of yttrium-90 may benefit some patients with osteoarthritis and persistent synovitis of large joints.

\section{Surgery}

The success of prosthetic joint replacements has greatly advanced management of end stage hip and knee osteoarthritis. Although issues relating to funding, waiting times, choice of prosthesis, and revision have to be faced, there is no doubt that such surgery can transform patients' lives. Other surgical approaches (arthroscopic lavage, osteotomy, and arthrodesis) may also be useful. The criteria for surgery are not definite but should probably include uncontrolled pain (particularly nocturnal pain) and severe impairment of function. Age, in itself, is not a contraindication.

\section{Psychological factors}

A major contribution to managing osteoarthritis has been the demonstration that a patient's psychological status (anxiety, depression, and social support) is an important determinant of symptomatic and functional outcome. Providing social support, even just regular telephone contact with a lay person, can effectively ameliorate symptoms. Perhaps the single most important help that any therapist can give is to emphasise to patients that osteoarthritis is not invariably progressive, that things can be done, and that the patients are not on their own.
The ABC of Rheumatology is edited by Michael L Snaith, senior lecturer in rheumatology at Nether Edge Hospital, Sheffield. 